\title{
Prevalence of and factors associated with renal dysfunction in rheumatoid arthritis patients: a cross-sectional study in community hospitals
}

\author{
Shunsuke Mori $^{1}$ (D) $\cdot$ Tamami Yoshitama $^{2} \cdot$ Naoyuki Hirakata $^{3} \cdot$ Yukitaka Ueki $^{3}$
}

Received: 28 June 2017 /Revised: 18 August 2017 / Accepted: 24 August 2017 / Published online: 7 September 2017

(C) The Author(s) 2017. This article is an open access publication

\begin{abstract}
This study was designed to determine the prevalence of renal dysfunction in rheumatoid arthritis (RA) patients and to identify factors associated with this complication. Between October 2014 and May 2015, we consecutively recruited RA patients at rheumatology sections of community hospitals in Japan. Each patient's absolute and body surface area (BSA)-indexed estimated glomerular filtration rate (eGFR) values were measured twice over a 3-month interval. Renal dysfunction was defined as absolute eGFR or BSAindexed eGFR $<60$. Albuminuria and hematuria were also recorded. Associations between renal dysfunction and possible risk factors were examined by multivariate logistic regression analyses. A total of 1908 outpatients with RA were included in this study. The prevalence of renal dysfunction based on absolute eGFR and BSA-indexed eGFR was 33.8 and $18.6 \%$, respectively. Albuminuria was observed in $8.1 \%$ of this patient cohort, and the prevalence of hematuria was $7.5 \%$. Advanced age (odds ratio [OR] 7.24, $p<0.001$ ), female sex (OR 3.12, $p<0.001$ ), hypertension (OR 2.22, $p<0.001$ ), and obesity (OR $0.59, p<0.001$ ) were independently associated with the risk of absolute eGFR-based renal dysfunction. Advanced age (OR 5.19, $p<0.001$ ) and hypertension (OR $3.05, p<0.001)$ also had associations with BSA-indexed
\end{abstract}

Shunsuke Mori

moris@ saisyunsou1.hosp.go.jp

1 Department of Rheumatology, Clinical Research Center for Rheumatic Diseases, NHO Kumamoto Saishunsou National Hospital, 2659 Suya, Kohshi, Kumamoto 861-1196, Japan

2 Yoshitama Clinic for Rheumatic Diseases, Kirishima, Kagoshima 899-5117, Japan

3 Rheumatic and Collagen Disease Center, Sasebo Chuo Hospital, Sasebo, Nagasaki 857-1195, Japan
eGFR-based renal dysfunction. RA duration, stages, severity, and cumulative steroid dose were considered significant risk factors in univariate analyses, but their associations were less potent after adjustment for other covariates. Renal dysfunction is relatively common in RA patients and is mainly associated with advanced age and hypertension but not with RA-related factors.

Keywords Glomerular filtration rate $\cdot$ Prevalence $\cdot$ Renal dysfunction $\cdot$ Rheumatoid arthritis $\cdot$ Risk factors

\section{Introduction}

Rheumatoid arthritis (RA) is an autoimmune disease in which persistent inflammatory changes occur in multiple synovial joints and many extra-articular organs. Renal involvement is one of the most important complications in the management of RA patients because of its prognostic value and therapeutic implications [1,2]. Although it has been reported that a considerable number of RA patients also have proteinuria, hematuria, and renal dysfunction, the figures reported for the prevalence and incidence of renal involvement vary depending on the criteria used to define the disease and the patient populations examined [1,3-8].

The factors associated with renal dysfunction in RA patients remain controversial. Previous studies of renal biopsy findings and clinicopathologic correlations showed that the most predominant pathological findings in clinical renal disease of RA patients were secondary amyloidosis, membranous nephropathy related to the use of antirheumatic drugs, mesangial glomerulonephritis possibly related to RA itself, and rheumatoid vasculitis commonly associated with rapidly progressive glomerulonephritis [9-11]. These studies were performed with RA patients who had received old antirheumatic drugs, such as gold, 
penicillamine, and bucillamine, which are known to be causative drugs for membranous nephropathy. These nephrotoxic drugs are, however, seldom used in current RA treatment. Secondary amyloidosis is now relatively rare in RA because of advances in the management and control of RA activity [12]. Although the contributory role of inflammation on renal dysfunction and renal damage was reported in previous studies on the general population [13-15], inconsistent results were obtained from recent studies on RA patients [5-8, 16-18].

The current scope of renal involvement in RA patients may widen. In daily practice, we encounter cases in which RA and renal disease coincide more often than we did in the past, despite the popularity of novel antirheumatic drugs, including biological agents, which can more effectively suppress the systemic inflammation associated with RA. The markedly increased risk of developing cardiovascular disease (CVD) has been recognized in RA patients for many years $[19,20]$, and this may contribute to the increased risk of premature death of this patient population [21-23]. Several studies have suggested that reduced renal function may be associated with CVD risk in RA patients [16, 17, 24, 25]. Appropriate risk management for impaired renal function is therefore important in reducing the risk of CVD mortality and improving outcomes of RA patients.

To determine the prevalence of renal dysfunction in RA patients, we performed a cross-sectional study at rheumatology sections of community hospitals in Japan. Each patient's absolute and body surface area (BSA)-indexed estimated glomerular filtration rate (eGFR) values were measured twice over a 3-month interval, and average values were then used to assess renal function. We also evaluated the independent associations of renal dysfunction with demographic and RArelated characteristics, the presence of traditional cardiovascular risk factors, and the use of RA medications.

\section{Patients and methods}

\section{Patients and study design}

Between October 2014 and May 2015, we consecutively recruited patients with RA from outpatient clinics for rheumatic diseases at the following community hospitals in Japan: NHO Kumamoto Saishunsou National Hospital, Yoshitama Clinic for Rheumatic Diseases, and Sasebo Chuo Hospital. Each patient was included once. Regardless of the reasons for their visits, eligible patients (namely, those who fulfilled the 1987 American College of Rheumatology [ACR] criteria or the 2010 ACR/European League Against Rheumatism [EULAR] criteria for diagnosis of RA) were enrolled in this study, unless they met any of the following exclusion criteria: being under 18 years of age, having acute kidney disease, receiving renal replacement therapy, or being pregnant.
For each patient, demographic characteristics, RA-related factors such as RA duration, Steinbrocker stage, clinical disease activity index (CDAI), health assessment questionnaire (HAQ), positivity of anti-cyclic citrullinated peptide antibodies (anti-CCP Abs), levels of serum C-reactive protein (CRP), and the current use of disease-modifying antirheumatic drugs (DMARDs), nonsteroidal anti-inflammatory drugs (NSAIDs), and steroids, together with traditional cardiovascular risk factors including hypertension, diabetes mellitus, smoking, body mass index (BMI), and levels of serum low-density lipoprotein cholesterol (LDL-C), were examined and recorded at inclusion. Serum levels of creatinine, together with the presence of albuminuria and hematuria, were also examined and recorded at the same time. Serum creatinine was measured twice over a 3-month interval for individual patients.

Hypertension was defined as systolic blood pressure of $140 \mathrm{mmHg}$ or greater, diastolic blood pressure of $90 \mathrm{mmHg}$ or greater, or the use of antihypertensive medications. Diabetes was defined as fasting blood glucose levels of $126 \mathrm{mg} / \mathrm{dl}$ or higher, serum HbAc1 higher than $6.0 \%$, or the use of diabetes medications. Hematuria was defined as the presence of five or more red blood cells per high-power field in three of three consecutive centrifuged specimens obtained at least 1 week apart. Urinary albumin was determined by a semi-quantitative urine dipstick testing kit (BM test MAU II, Roche Diagnostics K.K., Tokyo, Japan). Albuminuria was defined as " $2+$ " or more in dipstick testing, which corresponds to $50 \mathrm{mg} / \mathrm{l}$ or higher of urinary albumin.

NSAID intake was calculated according to the Assessment of Spondyloarthritis International Society (ASAS)-NSAID scoring system [26]. Information on the name and daily dose of NSAIDs as well as the number of days with NSAID intake during the previous 3 months was collected. To express results as a mean daily intake over the previous 3 months, the following formula was used: ASAS-NSAID score $=($ NSAID equivalent score $) \times($ days of intake during period of interest $) \times($ intake days per week) / (period of interest in days) [26]. Cumulative doses of methotrexate (MTX) and steroids (prednisolone equivalent) prescribed after initiation of RA treatment in the participating hospitals were also calculated for each user of these drugs. Information on weekly MTX dose, daily steroid dose, and duration of treatment was collected by reviewing patients' medical records.

\section{Renal function}

An eGFR was first calculated according to the following equation, which were developed for Japanese patients and officially approved by the Japanese Society of Nephrology: eGFR (ml/ $\left.\min / 1.73 \mathrm{~m}^{2}\right)=194 \times($ serum creatinine $[\mathrm{mg} /$ dl] $)^{-1.094} \times(\text { age })^{-0.287} \times 0.739$ (if female) [27]. A value calculated by this formula is an indexed eGFR that is adjusted to a standard BSA of $1.73 \mathrm{~m}^{2}$, but this indexation of eGFR can be 
misleading in populations with unusual anthropometric data, such as those containing obese or lean persons $[28,29]$. In the present study, the mean BSA value of participants was quite different from the standard BSA: it was $1.52 \mathrm{~m}^{2}$. An overestimation of eGFR may result if we use BSA-indexed values. Thus, we studied the prevalence and possible risk factors of renal dysfunction using both absolute eGFR values and BSAindexed eGFR values. The above-mentioned calculated value (BSA-indexed eGFR) was converted, using each patient's BSA, to an unindexed eGFR value (absolute eGFR): absolute eGFR $=$ indexed eGFR $\times$ patient's BSA $/ 1.73 \mathrm{~m}^{2}$ [30]. The BAS was calculated using the Du Bois formula: BSA $\left(\mathrm{m}^{2}\right)=0.007184 \times(\text { weight }[\mathrm{kg}])^{0.425} \times(\text { height }[\mathrm{cm}])^{0.725}$. Two measurements of eGFR were taken over a 3-month interval for individual patients, and average values were used in this study. Renal dysfunction was defined as absolute eGFR $<60 \mathrm{ml} / \mathrm{min}$ or BSA-indexed eGFR $<60 \mathrm{ml} / \mathrm{min} / 1.73 \mathrm{~m}^{2}$. eGFR stages were determined according to the Kidney Disease Improving Global Outcomes (KDIGO 2012) guidelines [31].

\section{Statistical analysis}

For comparisons of categorical variables between the groups with and without renal dysfunction, a statistical analysis was performed using the chi-square test. Continuous variables were assessed by the independent-measures $t$ test or the Mann-Whitney $U$ test. Multivariate logistic regression analysis was performed to evaluate the association between renal dysfunction as a dependent variable and a set of independent variables considered to be significant risk factors in univariate analyses. We also included, as independent variables, factors that were not significant in univariate analyses but could be confounding or clinically relevant variables. A backward stepwise selection procedure was used to select significant independent variables. The strength of association between renal dysfunction and these independent variables was estimated using odds ratios (ORs) and 95\% confidence intervals (95\% CIs). In addition, the receiver operating characteristic (ROC) curves and the corresponding area under the curve (AUC) values were calculated to provide an index of validity of multivariate logistic regression models.

For all tests, probability values ( $p$ values $)<0.05$ were considered to indicate statistical significance. All calculations were performed using PASW Statistics version 22 (SPSS Japan Inc., Tokyo, Japan).

\section{Results}

\section{Prevalence of renal dysfunction}

A total of 1908 patients with RA ( 350 men and 1558 women) were prospectively included in this study. Among these patients, 645 (33.8\%) were found to have renal dysfunction, defined as absolute eGFR $<60 \mathrm{ml} / \mathrm{min}$ (Table 1). The prevalence of renal dysfunction increased with age, affecting more than $50 \%$ of people over the age of 70 years, and it was higher in women (female $36.6 \%$ versus male $21.1 \%$ ). These trends were observed in all eGFR stages of renal dysfunction. Only a small percentage of patients $(2.1 \%)$ had severe or end-stage kidney disease defined as absolute eGFR $<30 \mathrm{ml} / \mathrm{min}$. The prevalence of albuminuria was $8.1 \%$ (155 out of 1908 patients), and among these patients, $58.7 \%$ were categorized as having renal dysfunction (Table 2). The prevalence of albuminuria was $5.1 \%$ (64 out of 1263 patients) in the group without renal dysfunction and $14.1 \%$ ( 91 out of 645 patients) in the renal dysfunction group. The prevalence of hematuria was $7.5 \%$ (143 out of 1908 patients), and among these patients, $48.3 \%$ had renal dysfunction. The prevalence of hematuria was $5.9 \%$ (74 out of 1263) in the group without renal dysfunction and $10.7 \%$ (69 out of 645 patients) in the renal dysfunction group.

When renal dysfunction was defined as BSA-indexed eGFR $<60 \mathrm{~min} / \mathrm{ml} / 1.73 \mathrm{~m}^{2}$, its prevalence was $18.6 \%$ (Table 1). Like the data obtained based on absolute eGFR values, the prevalence of BSA-indexed eGFR-based renal dysfunction was markedly higher in elderly patients, yet there was no significant difference between men and women. Similar results were observed in all eGFR stages of renal dysfunction. The rate of patients with severe or end-stage kidney disease defined as BSA-indexed eGFR $<30 \mathrm{ml} / \mathrm{min} /$ $1.73 \mathrm{~m}^{2}$ was $1.0 \%$. Among patients with albuminuria, $45.2 \%$ had renal dysfunction (Table 2). The prevalence of albuminuria was $5.5 \%$ (85 out of 1553 patients) in the group without renal dysfunction and $19.7 \%$ (39 out of 355 patients) in the renal dysfunction group. Among patients with hematuria, $27.3 \%$ had renal dysfunction. The prevalence of hematuria was $6.7 \%$ (104 out of 1553 patients) in the group without renal dysfunction and $11.0 \%$ (39 out of 355 patients) in the renal dysfunction group.

\section{Comparisons of demographic and clinical characteristics between RA patients with and without renal dysfunction based on absolute eGFR values}

Patients with renal dysfunction defined as absolute eGFR $<60 \mathrm{ml} /$ min were significantly older $(72.0$ versus 58.8 years, $p<0.001)$ and had longer RA duration (12.0 versus 10.1 years, $p<0.001$ ) (Table 3 ). This patient group was more often diagnosed as being at Steinbrocker classification stages III and IV (52.7 versus $45.2 \%, p=0.002$ ), and had a significantly higher mean level of HAQ $(0.56$ versus $0.35, p<0.001)$, but there was no significant difference in other RA-related indexes such as CDAI and anti-CCP positivity between the two patient groups. Renal dysfunction was significantly associated with female sex $(88.5$ versus $78.1 \%, p<0.001)$, but obesity 


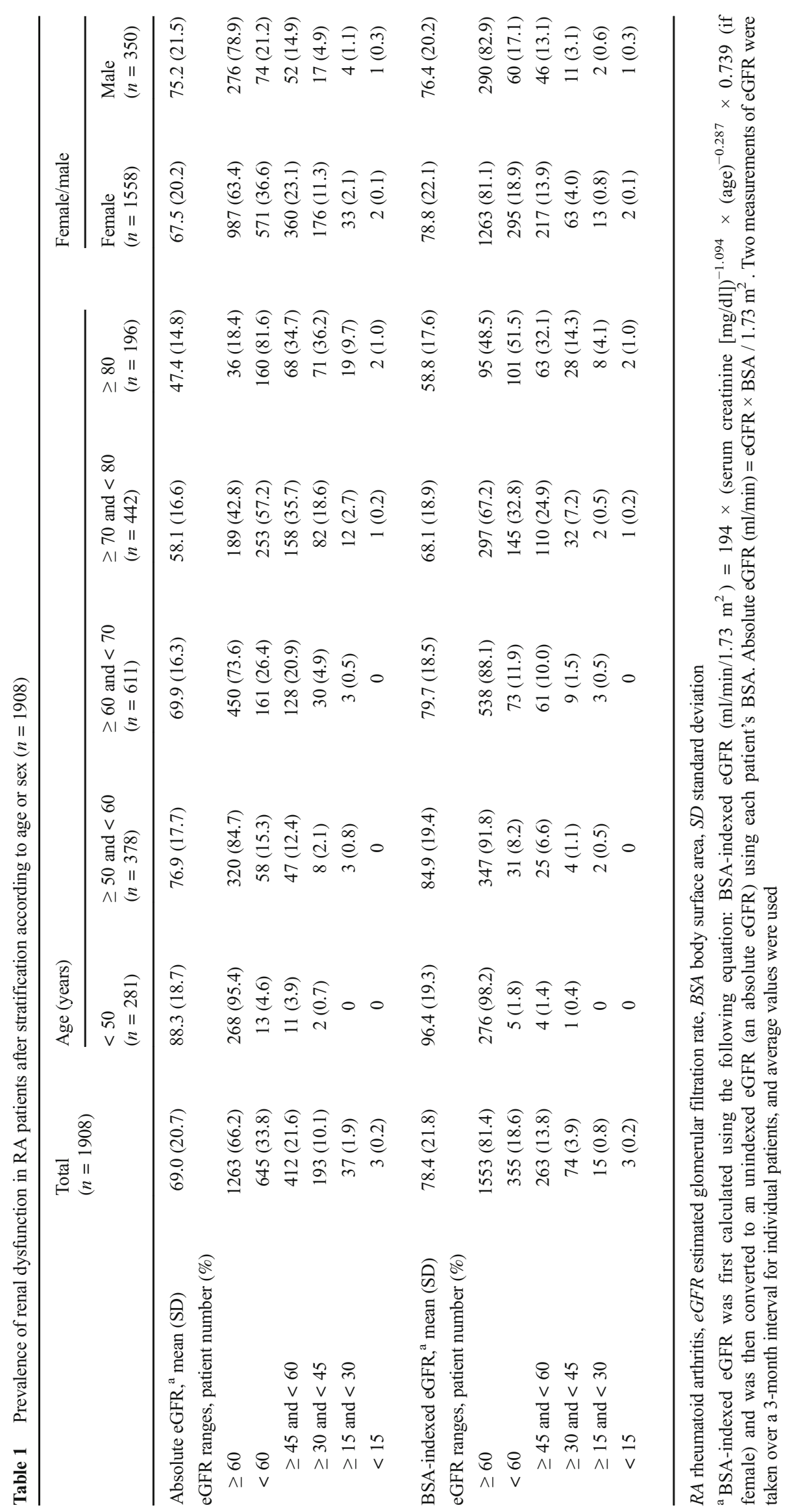


Table 2 Prevalence of renal dysfunction in RA patients after stratification according to the presence of albuminuria or hematuria $(n=1908)$

\begin{tabular}{|c|c|c|c|c|c|}
\hline & \multirow[t]{2}{*}{ Total $(n=1908)$} & \multicolumn{2}{|l|}{ Urine albumin } & \multicolumn{2}{|l|}{ Hematuria } \\
\hline & & $<" 2+"(n=1753)$ & $\geq " 2+"(n=155)$ & Negative $(n=1765)$ & Positive $(n=143)$ \\
\hline Absolute eGFR, ${ }^{\mathrm{a}}$ mean (SD) & $69.0(20.7)$ & $70.0(20.3)$ & $57.5(21.8)$ & $69.6(20.7)$ & $60.7(17.9)$ \\
\hline \multicolumn{6}{|l|}{ eGFR ranges, patient number $(\%)$} \\
\hline$\geq 60$ & $1263(66.2)$ & $1199(68.4)$ & $64(41.3)$ & $1189(67.4)$ & $74(51.7)$ \\
\hline$<60$ & $645(33.8)$ & $554(31.6)$ & $91(58.7)$ & $576(32.6)$ & $69(48.3)$ \\
\hline$\geq 45$ and $<60$ & $412(21.6)$ & $375(21.4)$ & $37(23.9)$ & $372(21.1)$ & $40(28.0)$ \\
\hline$\geq 30$ and $<45$ & $193(10.1)$ & $149(8.5)$ & $45(29.0)$ & $170(9.6)$ & $23(16.1)$ \\
\hline$\geq 15$ and $<30$ & $37(1.9)$ & $29(1.7)$ & $7(4.5)$ & $32(1.8)$ & $5(3.5)$ \\
\hline$<15$ & $3(0.2)$ & $1(0.1)$ & $2(1.3)$ & $2(0.1)$ & $1(0.7)$ \\
\hline BSA-indexed eGFR, ${ }^{a}$ mean (SD) & $78.4(21.8)$ & $79.5(21.3)$ & $65.5(22.4)$ & $79.0(21.8)$ & $70.2(19.3)$ \\
\hline \multicolumn{6}{|l|}{ eGFR ranges, patient number $(\%)$} \\
\hline$\geq 60$ & $1553(81.4)$ & $1468(83.7)$ & $85(54.8)$ & 1449 (82.1) & $104(72.7)$ \\
\hline$<60$ & 355 (18.6) & $285(16.3)$ & $70(45.2)$ & $316(17.9)$ & 39 (27.3) \\
\hline$\geq 45$ and $<60$ & $263(13.8)$ & $217(12.4)$ & $46(29.7)$ & $238(13.5)$ & $25(17.5)$ \\
\hline$\geq 30$ and $<45$ & 74 (3.9) & $57(3.3)$ & $17(11.0)$ & $63(3.6)$ & $11(7.7)$ \\
\hline$\geq 15$ and $<30$ & $15(0.8)$ & $10(0.6)$ & $5(3.2)$ & $13(0.7)$ & $2(1.4)$ \\
\hline$<15$ & $3(0.2)$ & $1(0.1)$ & $2(1.3)$ & $2(0.1)$ & $1(0.7)$ \\
\hline
\end{tabular}

$R A$ rheumatoid arthritis, $e G F R$ estimated glomerular filtration rate, $B S A$ body surface area, $S D$ standard deviation

${ }^{a}$ BSA-indexed eGFR was first calculated using the following equation: BSA-indexed eGFR $\left(\mathrm{ml} / \mathrm{min} / 1.73 \mathrm{~m}^{2}\right)=194 \times($ serum creatinine $[\mathrm{mg} / \mathrm{dl}])^{-1.094} \times(\mathrm{age})^{-0.287} \times 0.739$ (if female) and was then converted to an unindexed eGFR (an absolute eGFR) using each patient's BSA. Absolute eGFR $(\mathrm{ml} / \mathrm{min})=\mathrm{eGFR} \times \mathrm{BSA} / 1.73 \mathrm{~m}^{2}$. Two measurements of eGFR were taken over a 3-month interval for individual patients, and average values were used

$\left(\mathrm{BMI}>25 \mathrm{~kg} / \mathrm{m}^{2}\right.$ ) was less common in renal dysfunction patients $(16.6$ versus $23.8 \%, p<0.001)$. Hypertension and diabetes mellitus were more frequently observed in the renal dysfunction group (49.0 versus $24.4 \%, p<0.001 ; 12.7$ versus $8.9 \%, p=0.010$, respectively), but there was no significant difference in mean serum LDL-C concentrations. The rate of current or ex-smokers was lower in the renal dysfunction group (14.9 versus $24.2 \%, p<0.001$ ).

\section{Comparisons of demographic and clinical characteristics between RA patients with and without renal dysfunction based on BSA-indexed eGFR values}

Renal dysfunction defined as BSA-indexed eGFR $<60 \mathrm{ml} /$ $\mathrm{min} / 1.73 \mathrm{~m}^{2}$ was significantly associated with age (73.1 versus 61.0 years, $p<0.001$ ) but not with sex (Table 4$)$. RA duration was significantly longer in the renal dysfunction group (12.7 versus 10.3 years, $p<0.001)$, and this patient group showed a higher mean level of HAQ (0.59 versus $0.38, p<0.001)$. Anti-CCP positive rates were similar between the two patient groups. Hypertension and diabetes mellitus were more frequently present in the renal dysfunction group (60.1 versus $26.3 \%, p<0.001 ; 17.2$ versus $8.6 \%, p<0.001$, respectively), but there was no significant association of renal dysfunction with obesity
$\left(\mathrm{BMI}>25 \mathrm{~kg} / \mathrm{m}^{2}\right)$, smoking history, or serum LDL-C levels.

\section{Current use of RA medications in RA patients with and without renal dysfunction}

In terms of RA medications, $51.6 \%$ of RA patients were receiving MTX, and $33.6 \%$ were currently being treated with biological DMARDs (Table 3). NSAIDs and steroids were used in 25.6 and $30.7 \%$ of patients, respectively. The use of MTX was significantly less frequent in RA patients with renal dysfunction defined according to absolute eGFR values (43.6 versus $55.7 \%, p<0.001)$ and in those categorized with BSAindexed eGFR (39.4 versus 54.3\%, $p<0.001$ ) (Tables 3 and 4). The mean weekly MTX dose in users was also significantly lower in the group with absolute eGFR-based renal dysfunction (6.2 versus $8.2 \mathrm{mg} /$ week, $p<0.001)$ and in the group with BSA-indexed eGFR-based renal dysfunction (6.7 versus $8.4 \mathrm{mg} /$ week, $p<0.001$ ), but there was no significant difference in median cumulative MTX doses in users between the renal dysfunction group and the group without this complication. Biological agents were used more often in the group with absolute eGFR-based renal dysfunction (37.1 versus $31.8 \%$, $p=0.024)$. Steroid user rates were significantly higher in the group with BSA-indexed eGFR-based renal dysfunction (35.8 
Table 3 Demographic, clinical, and laboratory characteristics of RA patients with and without renal dysfunction based on absolute eGFR values

\begin{tabular}{|c|c|c|c|c|}
\hline & Total $(n=1908)$ & $\begin{array}{l}\text { With renal dysfunction } \\
(n=645)\end{array}$ & $\begin{array}{l}\text { Without renal dysfunction } \\
(n=1263)\end{array}$ & $p$ value $^{\mathrm{b}}$ \\
\hline Age, years, mean (SD) & $63.3(13.0)$ & $72.0(9.6)$ & $58.8(12.3)$ & $<0.001$ \\
\hline$\geq 65$ years, patient number $(\%)$ & $946(49.6)$ & $513(79.5)$ & $433(34.3)$ & $<0.001$ \\
\hline Male/female & $350 / 1558$ & $74 / 571$ & $276 / 987$ & $<0.001$ \\
\hline RA duration, years, mean (SD) & $10.8(9.5)$ & $12.0(10.7)$ & $10.1(8.8)$ & $<0.001$ \\
\hline$\leq 3$ years, patient number $(\%)$ & $400(21.0)$ & $114(17.7)$ & $286(22.6)$ & 0.012 \\
\hline Anti-CCP (+), patient number (\%) & $1486(77.9)$ & $503(78.0)$ & $983(77.8)$ & 0.95 \\
\hline Stage III/IV, patient number (\%) & $911(47.7)$ & $340(52.7)$ & $571(45.2)$ & 0.002 \\
\hline CDAI, mean (SD) & $6.2(6.7)$ & $6.3(6.4)$ & $6.2(6.8)$ & 0.91 \\
\hline HAQ, mean (SD) & $0.42(0.65)$ & $0.56(0.77)$ & $0.35(0.56)$ & $<0.001$ \\
\hline$\geq 1.0$, patient number $(\%)$ & $331(17.3)$ & $156(24.2)$ & $175(13.9)$ & $<0.001$ \\
\hline$<0.25$, patient number $(\%)$ & $671(35.2)$ & $278(43.1)$ & $393(31.1)$ & $<0.001$ \\
\hline Serum CRP, mg/dl, mean (SD) & $0.36(0.93)$ & $0.42(1.13)$ & $0.33(0.80)$ & 0.050 \\
\hline BMI, $\mathrm{kg} / \mathrm{m}^{2}$, mean (SD) & $22.5(3.7)$ & $22.1(3.2)$ & $22.8(3.9)$ & $<0.001$ \\
\hline$>25$, patient number $(\%)$ & $407(21.3)$ & $107(16.6)$ & $300(23.8)$ & $<0.001$ \\
\hline$<18.5$, patient number $(\%)$ & $196(10.3)$ & $70(10.9)$ & $126(10.0)$ & 0.58 \\
\hline Hypertension, patient number (\%) & $624(32.7)$ & $316(49.0)$ & $308(24.4)$ & $<0.001$ \\
\hline NIDDM, patient number $(\%)$ & $194(10.2)$ & $82(12.7)$ & $112(8.9)$ & 0.010 \\
\hline Serum LDL-C, mg/dl, mean (SD) & $111.4(28.8)$ & $110.7(29.7)$ & $111.8(28.4)$ & 0.45 \\
\hline$\geq 140 \mathrm{mg} / \mathrm{dl}$, patient number $(\%)$ & $274(14.4)$ & $103(16.0)$ & $171(13.5)$ & 0.17 \\
\hline Current/ex-smokers, number $(\%)$ & $402(21.1)$ & $96(14.9)$ & $306(24.2)$ & $<0.001$ \\
\hline Use of biologics, patient number $(\%)$ & $641(33.6)$ & $239(37.1)$ & $402(31.8)$ & 0.024 \\
\hline MTX use, patient number (\%) & 985 (51.6) & $281(43.6)$ & $704(55.7)$ & $<0.001$ \\
\hline Dose, mg/week, mean (SD) & $7.9(3.5)$ & $6.2(3.0)$ & $8.2(3.5)$ & $<0.001$ \\
\hline Cumulative dose, $\mathrm{g}$, median (IQR) & $2.8(1.2-4.7)$ & $2.8(1.2-4.4)$ & $2.7(1.2-4.8)$ & 0.19 \\
\hline NSAID use, patient number (\%) & $488(25.6)$ & $156(24.2)$ & $332(26.3)$ & 0.35 \\
\hline ASAS-NSAID score, mean (SD) & $48.5(21.0)$ & $49.4(20.0)$ & $48.1(21.5)$ & 0.52 \\
\hline Concurrent steroids, number (\%) & $237(12.4)$ & $81(12.6)$ & $156(12.4)$ & 0.88 \\
\hline Steroid use, patient number (\%) & $585(30.7)$ & $199(30.9)$ & $386(30.6)$ & 0.92 \\
\hline Dose, mg/day, mean (SD) & $3.0(2.1)$ & $2.9(1.8)$ & $3.0(2.2)$ & 0.49 \\
\hline Cumulative dose, $\mathrm{g}$, median (IQR) & $3.5(0.9-10.9)$ & $5.0(1.5-14.6)$ & $3.1(0.7-9.3)$ & $<0.001$ \\
\hline
\end{tabular}

$R A$ rheumatoid arthritis, $e G F R$ estimated glomerular filtration rate, anti-CCP anti-citrullinated peptide antibodies, $C D A I$ clinical disease activity index, $H A Q$ health assessment questionnaire, $C R P$ C-reactive protein, $B M I$ body mass index, NIDDM non-insulin-dependent diabetes mellitus, $L D L-C$ lowdensity lipoprotein cholesterol, MTX methotrexate, NSAIDs nonsteroidal anti-inflammatory drugs, ASAS Assessment of Spondyloarthritis International Society, $S D$ standard deviation, $I Q R$ interquartile range

${ }^{a}$ Defined as an absolute eGFR $<60 \mathrm{ml} / \mathrm{min}$. Average values of two measurements of eGFR taken over a 3-month interval were used

${ }^{\mathrm{b}}$ Comparison between RA patients with renal dysfunction and those without this complication

versus $29.5 \%, p=0.022$ ). Daily steroid doses in users were similar between the two groups, but the median cumulative dose in users was significantly higher in the absolute eGFRbased renal dysfunction group $(5.0$ versus $3.1 \mathrm{~g}, p<0.001)$ and in the BAS-indexed eGFR-based renal dysfunction group (5.0 versus $3.2 \mathrm{~g}, p<0.001$ ). There was no significant difference in rates of prescription of NSAIDs between the two groups in either the use of absolute eGFR or BAS-indexed eGFR for the definition of renal dysfunction. In addition, there were no significant differences in ASAS-NSAID scores or rates of concurrent steroid use with NSAIDs between both groups.

\section{Multivariate logistic regression analyses}

Results from multivariate logistic regression analyses are shown in Table 5. Age ( $\geq 65$ years), female sex, RA duration ( $\leq 3$ years), RA stages III and IV, HAQ $(\geq 1.0$ ), serum CRP levels, BMI (> 25), hypertension, NIDDM, serum LDL-C ( $\geq 140 \mathrm{mg} / \mathrm{dl}$ ), smoking history, biological DMARD use, NSAID use, and cumulative steroid dose $(\geq 5.5 \mathrm{~g})$ were selected as independent variables. We did not included MTX use or weekly MTX dose as a possible risk factor in analyses because the negative association seen in the univariate 
analyses was explained by the therapeutic avoidance of MTX by the treating rheumatologists. Regarding absolute eGFRbased renal dysfunction, advanced age (OR 7.24, $p<0.001$ ), female sex (OR 3.12, $p<0.001$ ), and hypertension (OR 2.22, $p<0.001)$ were the strong factors independently and positively associated with renal dysfunction in RA patients. Obesity (OR $0.59, p<0.001$ ) was the strong factor independently and negatively associated with renal dysfunction. High serum LDL-C levels, NSAID use, and high cumulative doses of steroid remained in the final model, but their associations were marginal.
Independent and strong associations of BSA-indexed eGFR-based renal dysfunction with advanced age (OR 5.19, $p<0.001$ ) and hypertension (OR 3.05, $p<0.001$ ) were confirmed (Table 5). Diabetes mellitus (OR 1.52, $p=0.022$ ) and high serum LDL-C levels (OR 1.53, $p=0.016$ ) were also significant factors associated with renal dysfunction but their associations were less potent after adjustment for other covariates. The association between renal dysfunction and high cumulative steroid doses was marginal. Female sex, RA duration, and serum CRP levels remained in the final model, but their associations were not significant.

Table 4 Demographic, clinical, and laboratory characteristics of RA patients with and without renal dysfunction based on BSA-indexed eGFR values

\begin{tabular}{|c|c|c|c|}
\hline & With renal dysfunction $^{\mathrm{a}}(n=355)$ & Without renal dysfunction $^{\mathrm{a}}(n=1553)$ & $p$ value $^{\mathrm{b}}$ \\
\hline Age, years, mean (SD) & $73.1(9.6)$ & $61.0(12.7)$ & $<0.001$ \\
\hline$\geq 65$ years, patient number $(\%)$ & $294(82.8)$ & $652(42.0)$ & $<0.001$ \\
\hline Male/female & $60 / 295$ & $290 / 1263$ & 0.49 \\
\hline RA duration, years, mean (SD) & $12.7(11.2)$ & $10.3(9.0)$ & $<0.001$ \\
\hline$\leq 3$ years, patient number $(\%)$ & $55(15.5)$ & $345(22.2)$ & 0.005 \\
\hline Anti-CCP $(+)$, patient number (\%) & $280(78.9)$ & $1206(77.7)$ & 0.67 \\
\hline Stage III/IV, patient number (\%) & $187(52.7)$ & $724(46.6)$ & 0.045 \\
\hline CDAI, mean (SD) & $6.3(6.7)$ & $6.2(6.6)$ & 0.84 \\
\hline HAQ, mean (SD) & $0.59(0.79)$ & $0.38(0.60)$ & $<0.001$ \\
\hline$\geq 1.0$, patient number $(\%)$ & $87(24.5)$ & $244(15.7)$ & $<0.001$ \\
\hline$<0.25$, patient number $(\%)$ & $157(44.2)$ & $514(33.1)$ & $<0.001$ \\
\hline Serum CRP, mg/dl, mean (SD) & $0.47(1.31)$ & $0.33(0.81)$ & 0.014 \\
\hline BMI, $\mathrm{kg} / \mathrm{m}^{2}$, mean (SD) & $22.7(3.4)$ & $22.5(3.7)$ & 0.31 \\
\hline$>25$, patient number $(\%)$ & $79(22.3)$ & $328(21.1)$ & 0.67 \\
\hline$<18.5$, patient number $(\%)$ & $29(8.2)$ & $167(10.8)$ & 0.18 \\
\hline Hypertension, patient number (\%) & $216(60.1)$ & $408(26.3)$ & $<0.001$ \\
\hline NIDDM, patient number $(\%)$ & $61(17.2)$ & $133(8.6)$ & $<0.001$ \\
\hline Serum LDL-C, mg/dl, mean (SD) & $112.4(31.3)$ & $111.2(28.2)$ & 0.50 \\
\hline$\geq 140 \mathrm{mg} / \mathrm{dl}$, patient number $(\%)$ & $61(17.4)$ & $213(13.6)$ & 0.094 \\
\hline Current/ex-smokers, number $(\%)$ & $64(18.0)$ & $338(21.8)$ & 0.13 \\
\hline Use of biologics, patient number $(\%)$ & $135(38.0)$ & $506(32.6)$ & 0.054 \\
\hline MTX use, patient number (\%) & $141(39.4)$ & $844(54.3)$ & $<0.001$ \\
\hline Dose, mg/week, mean (SD) & $6.7(3.0)$ & $8.4(3.6)$ & $<0.001$ \\
\hline Cumulative dose, g, median (IQR) & $2.7(1.3-4.3)$ & $2.8(1.2-4.7)$ & 0.22 \\
\hline NSAID use, patient number $(\%)$ & $91(25.6)$ & $397(25.6)$ & 1.00 \\
\hline ASAS-NSAID score, mean (SD) & $49.8(19.9)$ & $48.2(21.2)$ & 0.50 \\
\hline Concurrent steroids, number (\%) & $54(15.2)$ & $183(11.8)$ & 0.094 \\
\hline Steroid use, patient number (\%) & $127(35.8)$ & $458(29.5)$ & 0.022 \\
\hline Dose, mg/day, mean (SD) & $3.0(1.9)$ & $2.9(2.1)$ & 0.80 \\
\hline Cumulative dose, g, median (IQR) & $5.0(1.5-14.6)$ & $3.2(0.8-9.5)$ & $<0.001$ \\
\hline
\end{tabular}

$R A$ rheumatoid arthritis, $e G F R$ estimated glomerular filtration rate, anti-CCP anti-citrullinated peptide antibodies, $C D A I$ clinical disease activity index, $H A Q$ health assessment questionnaire, $C R P$ C-reactive protein, $B M I$ body mass index, $N I D D M$ non-insulin-dependent diabetes mellitus, $L D L-C$ lowdensity lipoprotein cholesterol, MTX methotrexate, NSAIDs nonsteroidal anti-inflammatory drugs, ASAS Assessment of Spondyloarthritis International Society, $S D$ standard deviation, $I Q R$ interquartile range

${ }^{\text {a }}$ Defined as a BSA-indexed eGFR $<60 \mathrm{ml} / \mathrm{min} / 1.73 \mathrm{~m}^{2}$. Average values of two measurements of eGFR taken over a 3-month interval were used

${ }^{\mathrm{b}}$ Comparison between RA patients with renal dysfunction and those without 


\section{Discussion}

In the present study, the prevalence of renal dysfunction defined as absolute eGFR $<60 \mathrm{ml} / \mathrm{min}$ was $33.8 \%$ in RA patients, whereas $18.6 \%$ had renal dysfunction defined as BSAindexed eGFR $<60 \mathrm{ml} / \mathrm{min} / 1.73 \mathrm{~m}^{2}$. Albuminuria and hematuria were observed in 8.1 and $7.5 \%$ of RA patients in our cohort, respectively. In multivariate regression analyses, advanced age and hypertension were found to be the strong factors independently associated with the risk of renal dysfunction when it was defined using either absolute eGFR or BSA-indexed eGFR values. The strong association of female sex (positive association) and obesity (negative association) was observed only with absolute eGFR-based renal dysfunction. Diabetes mellitus, high serum LDL-C levels, and high cumulative steroid doses were significantly associated with renal dysfunction, but these associations were less potent than advanced age and hypertension.

Few studies have determined the prevalence of renal dysfunction defined according to eGFR data in RA patients. A recent study using a nationwide database of rheumatic diseases in Japan (the NinJa study) showed that the prevalence of BSA-indexed eGFR $\geq 30$ and $<60 \mathrm{ml} / \mathrm{min} / 1.73 \mathrm{~m}^{2}, \geq 15$ and $<30 \mathrm{ml} / \mathrm{min} / 1.73 \mathrm{~m}^{2}$, and $<15 \mathrm{ml} / \mathrm{min} / 1.73 \mathrm{~m}^{2}$ was 17.5 , 0.8 , and $0.2 \%$, respectively, which was very similar to the data obtained in the present study [32]. In a nationwide, large-scale epidemiological study for the Japanese general population, Imai et al. showed that the prevalence of renal dysfunction defined as BSA-indexed eGFR $<60 \mathrm{ml} / \mathrm{min} / 1.73 \mathrm{~m}^{2}$ was $20.5 \%$ [33]. This is similar to the prevalence in the RA cohort of the present study and in that of the NinJa study (18.6\% in both studies). In studies from Western countries, the prevalence of renal dysfunction defined as BSA-indexed eGFR $<60 \mathrm{ml} / \mathrm{min} / 1.73 \mathrm{~m}^{2}$ in RA patients was reported to be 8.8\% (the UK), 12.8\% (France), and 15.0\% (France) [4, 5, 8]. Hickson et al. reported in a follow-up study for an inception RA cohort in the USA that the 20-year cumulative incidence of renal dysfunction was higher in RA patients than in non-RA participants ( 25 versus. $20 \%, p=0.03$ ) [7].

The aforementioned studies used BSA-indexed eGFR values in determining the prevalence of renal dysfunction; however, indexing of eGFR for BSA can have a substantial impact on clinical studies involving patients with extreme body sizes, namely, an underestimation of eGFR in the case of obese patients and an overestimation of GFR in the case of underweight patients $[28,29]$. In the present study, the mean absolute eGFR value was significantly lower than that of BSA-indexed eGFR values $(69.0 \mathrm{ml} / \mathrm{min}$ versus $78.4 \mathrm{ml} /$ $\min / 1.73 \mathrm{~m}^{2}$ ), and the rate of patients with absolute eGFRbased renal dysfunction was nearly twofold higher than the rate of patients with renal dysfunction determined based on BSA-indexed eGFR values (33.8 versus $18.6 \%$ ). These results can be explained by the fact that a considerable number of participants had extremely low BSA values (mean, $1.52 \mathrm{~m}^{2}$ ). Considering that RA patients, especially elderly individuals, are likely to present a smaller body size than $1.73 \mathrm{~m}^{2}$, it would be better to consider using absolute eGFR instead of BSAindexed eGFR in studies for RA patients. The Chronic Kidney Disease Epidemiology Collaboration (CKD-EPI) equation and the Modification of Diet in Renal Disease (MDRD) equation are currently the most widely used methods for the estimation of BSA-indexed eGFR in clinical practice as well as clinical studies. Recently, Redal-Baigorri et al. showed that the performance of these formulas could be significantly improved in the assessment of individual kidney function if absolute values are used by removing the BSA normalization factor. In that study, absolute eGFR by CKD-EPI is comparable to measured GFR [30].

Over the last few years, the causative role of inflammation in the development of renal dysfunction and disease has been the subject of controversy. Two prospective follow-up studies on patients with inflammatory rheumatic diseases showed that the severity of psoriasis and the presence of psoriatic arthritis

Table 5 Factors associated with renal dysfunction in RA patients

\begin{tabular}{lrrr}
\hline & OR & $95 \%$ CI & $p$ value \\
\hline Absolute eGFR-based renal dysfunction & & & \\
Age $(\geq 65$ years $)$ & 7.24 & $5.71-9.18$ & $<0.001$ \\
Female sex & 3.12 & $2.29-4.24$ & $<0.001$ \\
BMI $(>25)$ & 0.59 & $0.44-0.78$ & $<0.001$ \\
Hypertension & 2.22 & $1.76-2.80$ & $<0.001$ \\
Serum LDL-C $(\geq 140 \mathrm{mg} / \mathrm{dl})$ & 1.38 & $1.01-1.89$ & 0.046 \\
NSAID use & 0.77 & $0.60-1.00$ & 0.050 \\
Cumulative steroid dose $(\geq 5.5 \mathrm{~g})$ & 1.40 & $1.00-1.95$ & 0.049 \\
BSA-indexed eGFR-based renal dysfunction & & \\
Age ( $\geq$ 65 years) & 5.19 & $3.83-7.05$ & $<0.001$ \\
Female sex & 1.38 & $0.99-1.92$ & 0.061 \\
RA duration $(\leq 3$ years) & 0.72 & $0.51-1.01$ & 0.058 \\
Serum CRP levels & 1.12 & $0.99-1.27$ & 0.068 \\
Hypertension & 3.05 & $2.37-3.96$ & $<0.001$ \\
NIDDM & 1.52 & $1.06-2.18$ & 0.022 \\
Serum LDL-C $(\geq 140 \mathrm{mg} / \mathrm{dl})$ & 1.53 & $1.08-2.17$ & 0.016 \\
Cumulative steroid dose $(\geq 5.5 \mathrm{~g})$ & 1.45 & $1.01-2.08$ & 0.047 \\
\hline
\end{tabular}

Two separate multivariate logistic regression analyses were conducted to evaluate factors associated with the risk of renal dysfunction based on absolute eGFR and BSA-indexed eGFR. Independent factors that remained in the final models are shown. The final step yielded an AUC-ROC of 0.79 (95\% CI $0.77-0.81, p<0.001$ ) for absolute eGFRbased renal dysfunction and $0.78(95 \%$ CI $0.76-0.81, p<0.001)$ for BSA-indexed eGFR-based renal dysfunction

$R A$ rheumatoid arthritis, $e G F R$ estimated glomerular filtration rate, $B M I$ body mass index, $L D L-C$ low-density lipoprotein cholesterol, NSAID nonsteroidal anti-inflammatory drug, $B S A$ body surface area, $C R P \mathrm{C}$ reactive protein, $N I D D M$ non-insulin-dependent diabetes mellitus, $O R$ odds ratio, $95 \%$ CI 95\% confidence interval, $A U C$ area under the curve, $R O C$ receiver operating characteristics 
were significant factors associated with CKD [34, 35]. Regarding RA patients, in a 5-year follow-up study, Chiu et al. showed that, after adjusting for traditional cardiovascular risk factors, RA patients still had a higher risk of developing CKD compared with individuals without RA, and they suggested that chronic inflammation may be one of the main factors that contributed to the increased CKD risk in RA patients [17]. In a retrospective observational study, Kochi et al. showed that, even after adjusting for classical CKD risk factors, a persistently high level of CRP remained a significant risk factor for the development of CKD in RA patients [18]. In contrast, Daoussis et al. showed that renal dysfunction is not related to RA-related factors, with the exception of the presence of extra-articular complications, including disease activity and duration, disability, and the use of RA medications [5]. Couderc et al. also revealed that neither disease activity nor severity of RA is associated with renal impairment [8]. Haroon et al. indicated that, in patients with RA or seronegative inflammatory arthritis, no association was noted between renal dysfunction and the use of inflammatory markers, duration of arthritis, or DMARDs [16].

In the present study, traditional cardiovascular risk factors, such as advanced age and hypertension, were identified as the factors independently associated with renal dysfunction in RA patients. Several studies also identified some traditional cardiovascular risk factors as being independently associated with the risk of developing renal dysfunction in RA patients $[5,7,8,16$, 17]. Considering recent advances in the control of RA disease activity and the decreased frequency of nephrotoxic DMARD use, the impact of RA-related factors may have become limited. Instead, it may be traditional cardiovascular risk factors that mainly contribute to the development of renal damage in RA patients. The prevalence of traditional risk factors is apparently increasing in the general population, which may be the cause of the increased prevalence of decreased renal function [36]. This seems to be true for RA patients.

NSAIDs have been considered to cause renal damage. Chiu et al. showed that NSAIDs are associated with an increased risk of CKD in RA patients [17]. In the present study, however, the use of NSAIDs was not identified as a factor associated with renal dysfunction in RA patients, and there was no significant difference in ASAS-NSAID scores between the groups with and without renal dysfunction. Similarly, Couderc et al. revealed that there was no significant association between renal dysfunction and the use of NSAIDs in RA patients. ASAS-NSAID scores did not differ between the two groups [8]. Moller et al. recently showed in a longterm prospective study that chronic use of NSAIDs had no negative impact on renal function estimates for RA patients with moderate renal dysfunction, defined as eGFR $<60$ and $\geq 30 \mathrm{ml} / \mathrm{min}$ [37]. Several studies indicated that NSAID use was significantly associated with a higher GFR compared with nonusers and suggested that NSAIDs may have been prescribed or taken by patients with better renal function at baseline $[5,7,16]$.

Our findings are subject to at least two limitations. First, the design of the present study was cross-sectional, which cannot provide any indication as to the sequence of events. Even when strong statistical evidence indicates that a variable is an independent risk factor for an outcome, this does not necessarily indicate that the risk factor causally contributes to the outcome [38]. Therefore, it was difficult to make a causal inference. Second, we used the eGFR estimating equations instead of direct measurements of GFR using a clearance of exogenous filtration markers. Muscle wasting is a common feature in RA patients, and since serum creatinine levels are influenced by muscle mass, eGFR values calculated by using serum creatinine levels may overestimate renal function in RA patients. In clinical practice, however, the direct measurements of GFR are impractical because they are expensive and time consuming.

In conclusion, renal dysfunction is common in RA patients. When determined according to BSA-indexed eGFR, its prevalence is comparable with that in the general population of Japan. However, the use of absolute eGFR may be a better choice for the evaluation of the renal function of RA patients. Advanced age and hypertension are the strong and independent factors associated with renal dysfunction in this patient population, while the association of RA-related factors with renal dysfunction appears to be less potent. This may be explained by recent advances in the management and control of systemic inflammation associated with RA. Regular monitoring of renal function should be implemented, especially for elderly RA patients with hypertension. Identification of the high-risk group for renal dysfunction can not only facilitate early intervention to prevent this complication but can also help to achieve optimal management of RA patients.

Funding information This study was supported by research funds from the National Hospital Organization, Japan.

Compliance with ethical standards This study was conducted in accordance with the principles of the Declaration of Helsinki (2008). The protocol of this study also meets the requirements of the Ethical Guidelines for Medical and Health Research Involving Human Subjects, Japan (2014) and has been approved by the Human Research Ethics Committee of NHO Kumamoto Saishunsou National Hospital (No. 2615). Patient informed consent to participate was obtained.

\section{Disclosures None.}

Open Access This article is distributed under the terms of the Creative Commons Attribution 4.0 International License (http:// creativecommons.org/licenses/by/4.0/), which permits unrestricted use, distribution, and reproduction in any medium, provided you give appropriate credit to the original author(s) and the source, provide a link to the Creative Commons license, and indicate if changes were made. 


\section{References}

1. Sihvonen S, Korpela M, Mustonen J, Laippala P, Pasternack A (2004) Renal disease as a predictor of increased mortality among patients with rheumatoid arthritis. Nephron Clin Pract 96:c107-c114

2. Mori S, Yoshitama T, Hidaka T, Hirakata N, Ueki Y (2015) Effectiveness and safety of tocilizumab therapy for patients with rheumatoid arthritis and renal insufficiency: a real-life registry study in Japan (the ACTRA-RI study). Ann Rheum Dis 74:627-630

3. Koseki Y, Terai C, Moriguchi M, Uesato M, Kamatani N (2001) A prospective study of renal disease in patients with early rheumatoid arthritis. Ann Rheum Dis 60:327-331

4. Karie S, Gandjbakhch F, Janus N et al (2008) Kidney disease in RA patients: prevalence and implication on RA-related drugs management: the MATRIX study. Rheumatology (Oxford) 47:350-354

5. Daoussis D, Panoulas VF, Antonopoulos I et al (2010) Cardiovascular risk factors and not disease activity, severity or therapy associate with renal dysfunction in patients with rheumatoid arthritis. Ann Rheum Dis 69:517-521

6. Daoussis D, Panoulas VF, John $\mathrm{H}$ et al (2011) Microalbuminuria in rheumatoid arthritis in the post penicillamine/gold era: association with hypertension, but not therapy or inflammation. Clin Rheumatol 30:477-484

7. Hickson LJ, Crowson CS, Gabriel SE, McCarthy JT, Matteson EL (2014) Development of reduced kidney function in rheumatoid arthritis. Am J Kidney Dis 63:206-213

8. Couderc M, Tatar Z, Pereira B et al (2016) Prevalence of renal impairment in patients with rheumatoid arthritis: results from a cross-sectional multicenter study. Arthritis Care Res (Hoboken) 68:638-644

9. Boers M, Croonen AM, Dijkmans BA et al (1987) Renal findings in rheumatoid arthritis: clinical aspects of 132 necropsies. Ann Rheum Dis 46:658-663

10. Helin HJ, Korpela MM, Mustonen JT, Pasternack AI (1995) Renal biopsy findings and clinicopathologic correlations in rheumatoid arthritis. Arthritis Rheum 38:242-247

11. Nakano M, Ueno M, Nishi S et al (1998) Analysis of renal pathology and drug history in 158 Japanese patients with rheumatoid arthritis. Clin Nephrol 50:154-160

12. Immonen $\mathrm{K}$, Finne $\mathrm{P}$, Gronhagen-Riska $\mathrm{C}$ et al (2011) A marked decline in the incidence of renal replacement therapy for amyloidosis associated with inflammatory rheumatic diseases - data from nationwide registries in Finland. Amyloid 18:25-28

13. Stuveling EM, Hillege HL, Bakker SJ, Gans RO, De Jong PE, De Zeeuw D (2003) C-reactive protein is associated with renal function abnormalities in a non-diabetic population. Kidney Int 63:654-661

14. Fried L, Solomon C, Shlipak M et al (2004) Inflammatory and prothrombotic markers and the progression of renal disease in elderly individuals. J Am Soc Nephrol 15:3184-3191

15. Tonelli M, Sacks F, Pfeffer M et al (2005) Biomarkers of inflammation and progression of chronic kidney disease. Kidney Int 68: 237-245

16. Haroon M, Adeeb F, Devlin J, O Gradaigh D, Walker F (2011) A comparative study of renal dysfunction in patients with inflammatory arthropathies: strong association with cardiovascular diseases and not with anti-rheumatic therapies, inflammatory markers or duration of arthritis. Int J Rheum Dis 14:255-260

17. Chiu HY, Huang HL, Li CH et al (2015) Increased risk of chronic kidney disease in rheumatoid arthritis associated with cardiovascularcomplications - a national population-based cohort study. PLoS One 10:e136508

18. Kochi M, Kohagura K, Shiohira Y, Iseki K, Ohya Y (2016) Inflammation as a risk of developing chronic kidney disease in rheumatoid arthritis. PLoS One 11:e0160225
19. Nurmohamed MT, Heslinga M, Kitas GD (2015) Cardiovascular comorbidity in rheumatic diseases. Nat Rev Rheumatol 11:693-704

20. Hollan I, Dessein PH, Ronda N et al (2015) Prevention of cardiovascular disease in rheumatoid arthritis. Autoimmun Rev 14:952-969

21. Solomon DH, Karlson EW, Rimm EB et al (2003) Cardiovascular morbidity and mortality in women diagnosed with rheumatoid arthritis. Circulation 107:1303-1307

22. Gabriel SE (2008) Cardiovascular morbidity and mortality in rheumatoid arthritis. Am J Med 121:S9-14

23. Avina-Zubieta JA, Choi HK, Sadatsafavi M, Etminan M, Esdaile JM, Lacaille D (2008) Risk of cardiovascular mortality in patients with rheumatoid arthritis: a meta-analysis of observational studies. Arthritis Rheum 59:1690-1697

24. van Sijl AM, van den Oever IA, Peters MJ et al (2012) Subclinical renal dysfunction is independently associated with cardiovascular events in rheumatoid arthritis: the CARRE study. Ann Rheum Dis 71:341-344

25. Dessein PH, Hsu HC, Tsang L et al (2015) Kidney function, endothelial activation and atherosclerosis in black and white Africans with rheumatoid arthritis. PLoS One 10:e0121693

26. Dougados M, Simon P, Braun J et al (2011) ASAS recommendations for collecting, analysing and reporting NSAID intake in clinical trials/epidemiological studies in axial spondyloarthritis. Ann Rheum Dis 70:249-251

27. Matsuo S, Imai E, Horio $M$ et al (2009) Revised equations for estimated GFR from serum creatinine in Japan. Am J Kidney Dis 53:982-992

28. Delanaye P, Mariat C, Cavalier E, Krzesinski JM (2009) Errors induced by indexing glomerular filtration rate for body surface area: reductio ad absurdum. Nephrol Dial Transplant 24:3593-3596

29. Redal-Baigorri B, Rasmussen K, Heaf JG (2014) Indexing glomerular filtration rate to body surface area: clinical consequences. J Clin Lab Anal 28:83-90

30. Redal-Baigorri B, Rasmussen K, Heaf JG (2013) The use of absolute values improves performance of estimation formulae: a retrospective cross sectional study. BMC Nephrol 14:271

31. Levin A, Stevens PE (2014) Summary of KDIGO 2012 CKD Guideline: behind the scenes, need for guidance, and a framework for moving forward. Kidney Int 85:49-61

32. Saisho K, Yoshikawa N, Sugata K, Hamada H, Tohma S (2016) Prevalence of chronic kidney disease and administration of RArelated drugs in patients with RA: the NinJa 2012 study in Japan. Mod Rheumatol 26:331-335

33. Imai E, Horio M, Iseki K et al (2007) Prevalence of chronic kidney disease (CKD) in the Japanese general population predicted by the MDRD equation modified by a Japanese coefficient. Clin Exp Nephrol 11:156-163

34. Chi CC, Wang J, Chen YF, Wang SH, Chen FL, Tung TH (2015) Risk of incident chronic kidney disease and end-stage renal disease in patients with psoriasis: a nationwide population-based cohort study. J Dermatol Sci 78:232-238

35. Chiu HY, Huang HL, Li CH et al (2015) Increased risk of glomerulonephritis and chronic kidney disease in relation to the severity of psoriasis, concomitant medication, and comorbidity: a nationwide population-based cohort study. Br J Dermatol 173:146-154

36. Domrongkitchaiporn S, Sritara P, Kitiyakara C et al (2005) Risk factors for development of decreased kidney function in a southeast Asian population: a 12-year cohort study. J Am Soc Nephrol 16: 791-799

37. Moller B, Pruijm M, Adler S et al (2015) Chronic NSAID use and long-term decline of renal function in a prospective rheumatoid arthritis cohort study. Ann Rheum Dis 74:718-723

38. Brotman DJ, Walker E, Lauer MS, O'Brien RG (2005) In search of fewer independent risk factors. Arch Intern Med 165:138-145 